This is a postprint version of the following published document:

Espasa, Antoni; Sargan, J. D. The spectral estimation of simultaneous equation systems with lagged endogenous variables. International Economic Review, Oct. 1977, vol. 18, no 3, p. 583-605. Available in http://dx.doi.org/10.2307/2525950

(C) Wiley 


\title{
THE SPECTRAL ESTIMATION OF SIMULTANEOUS EQUATION SYSTEMS WITH LAGGED ENDOGENOUS VARIABLES
}

\author{
By Antoni Espasa ${ }^{1}$ and J. Denis Sargan
}

\section{INTRODUCTION}

Spectral estimators for the structural parameters of the simultaneous equations model with a general stationary structure (GSS) for the disturbances have been proposed by Hannan and Terrell [7] and further work has been done by one of the present authors [1]. In this paper we extend the mentioned spectral method (Specfiml) to be the estimation of a model containing lagged endogenous variables and we compare its asymptotic properties with those of time domain methods. The plan of the paper is as follows: in Section 2 we set up the notation and assumptions used later. In Section 3 we discuss alternative likelihood functions and their first order conditions and a three stage least squares (3SLS) approximation is proposed. Section 4 discusses the asymptotic properties. Section 5 illustrates the computation of the estimators and in Section 6 we show that as the order of the autoregressive (AR) error process, assumed in an autoregressive full information maximum likelihood estimation, tends to infinity, the asymptotic variance matrix (AVM) for that case tends to the AVM of the Specfiml estimators.

\section{ASSUMPTIONS AND NOTATION}

The notation used in this paper follows closely that used in Espasa [1], and to facilitate reading we list here some of the terminology.

We use small letters to represent scalars and small letters underlined by tilde for column vectors; the transposition sign (') is used to denote a row vector. Matrices will be represented by capital letters. The exceptions to these rules will be made clear in the text.

The Kronecker product between two matrices $A$ and $B$ is defined as $A \otimes B$ $=\left(a_{i j} B\right)$. Vec is an operator that transforms a matrix into a vector obtained by putting the columns of the matrix one below the other. We use $\bar{A}$ for the complex conjugate and $A^{*}$ for the transpose of the complex conjugate of the matrix A.

For the derivation of the spectral estimators it is useful to transform the model by a unitary transformation and we do this by applying the matrix

1 The first author, now working at the Banco de España, Madrid, Spain, wishes to acknowledge financial help from the British Council and Fundación Juan March. 


$$
Q=\left\{\frac{1}{\sqrt{T}}[\exp (2 \pi i j t / T)]\right\}, \quad j, t=0, \ldots, T-1
$$

where $T$ is the number of observations, to it. Using

$$
\omega_{j}=\frac{2 \pi j}{T} \quad j=0,1, \ldots, T-1
$$

the rows of $Q$ can be written as

$$
q^{\prime}\left(\omega_{j}\right)=\left\{\frac{1}{\sqrt{T}}\left[\exp \left(i \omega_{j} \cdot 0\right) \exp \left(i \omega_{j} \cdot 1\right) \cdots \exp \left(i \omega_{j}(T-1)\right)\right]\right\} .
$$

For $T$ we use

$$
T=2 M m,
$$

and for the ease of exposition we assume $m$ is odd. Then the $\omega_{j}$ 's can be grouped into $2 M$ sets the $h^{\text {th }}$ of which consists of $m$ adjacent $\omega_{j}$ 's clustered around

$$
\lambda_{h}=0+\frac{\pi h}{M}, \quad h=0, \ldots, 2 M-1
$$

and we call $Q\left(\lambda_{h}\right)$ the submatrix from $Q$ corresponding to the $h^{\text {th }}$ set. Note that for the zero set the $Q\left(\lambda_{0}\right)$ matrix is obtained taking the $(m-1) / 2$ last rows and the $(m+1) / 2$ first rows of $Q$. The assumption of the same $m$ in each set is by no means crucial and can be easily relaxed.

Suppose $X$ is a $T \times k$ matrix of $T$ observations on $k$ variables, then the Fourier transform of $X$ at the frequency $\omega_{j}$ is

$$
w_{X}^{\prime}\left(\omega_{j}\right)=\underline{q}^{\prime}\left(\omega_{j}\right) X .
$$

The matrix of Fourier transforms corresponding to the $h^{\text {th }}$ set is given by

$$
W_{X}\left(\lambda_{h}\right)=Q\left(\lambda_{h}\right) X,
$$

and the matrix corresponding to the all $2 M$ sets is given by

$$
W_{X}=Q X \text {. }
$$

Then the smoothed or average periodogram is

$$
I_{X}\left(\lambda_{h}\right)=\frac{1}{2 \pi m} W_{X}^{*}\left(\lambda_{h}\right) W_{X}\left(\lambda_{h}\right) .
$$

Note that $I_{X}\left(\lambda_{(2 M-h)}\right)=I_{X}\left(\lambda_{h}\right)^{*}$. The crossperiodogram between two sets of variables $Y$ and $Z$ is defined in a similar way. ${ }^{2}$

The spectral density matrices are denoted by $F(\lambda)$ with the appropriate variable

${ }^{2}$ Note that this gives a definition of the spectral density matrix which is the conjugate of that used by most authors, notably Hannan [4]. Our convention leads to a more natural form for expressions such as (6) or (17) below, and has no effect on the real form of the likelihood function or the likelihood estimates. 
suffixes, in a way similar to the periodogram.

The model, the parameters of which we want to estimate, can be expressed as

$$
B_{0} Y^{\prime}+B_{1} Y_{1}^{\prime}+\cdots+B_{r} Y_{r}^{\prime}+\Gamma Z^{\prime}=U^{\prime}
$$

or using

$$
A=\left[B_{0} B_{1} \cdots B_{r} \Gamma\right]=\left[B_{0} \Gamma^{\dagger}\right]
$$

and

$$
X=\left[Y Y_{1} \cdots Y_{r} Z\right]=\left[Y Y^{\dagger} Z\right]=\left[Y Z^{\dagger}\right]
$$

as

$$
A X^{\prime}=U^{\prime}
$$

where $Y$ is the $(T \times p)$ observation matrix of the endogenous variables, $Y_{s}$ is the $Y$ matrix lagged $s$ periods, $Z$ is a $(T \times k)$ observation matrix of the exogenous variables, $U$ is the matrix of unknown disturbances and $A$ the coefficient matrix.

For the vector error process $\left\{u_{t}\right\}$ we assume this to be a purely non-deterministic process with a finite variance, which can be represented as

$$
{\underset{\sim}{u}}_{t}=\sum_{j=0}^{\infty} A(j) \varepsilon_{t-j}, \quad A(0)=I,
$$

and that $\sum_{j=0}^{\infty} j\|A(j)\|<\infty$,

where the $\varepsilon_{t-j}$ are i.i.d., random error vectors of dimension $p$ with zero mean and non-singular covariance matrix $G .\|A(j)\|$ is a suitable matrix norm.

The spectral density of $u_{t}$ is of the form

$$
F_{U}(\lambda)=\frac{1}{2 \pi}\left[\sum_{j} A(j) e^{-i j \lambda}\right] G\left[\sum_{j} A(j) e^{-i j \lambda}\right]^{*},
$$

and we require that

$$
\operatorname{det} F_{U}(\lambda)>0, \quad \lambda \in[-\pi, \pi] .
$$

For the asymptotic theory presented below we need consistent estimators of $F_{U}(\lambda)$ and for that purpose we require that $T$ and $M$ tend to infinity and also $M^{2} / T \rightarrow 0$. The asymptotic theory involved here can be presented heuristically in the following way. We assume that the spectra of the disturbances can be divided in $2 M$ bands between $[-\pi, \pi]$ such that for each band $F_{U}(\lambda)$ is constant. Then we let first $T$ tend to infinity and then we allow $M$ to tend to infinity. As $T$ tends to infinity, the number of parameters in the likelihood initially remains finite, but when we also allow $M$ to tend to infinity, the block diagonal matrix

$$
F_{U}=\left(\begin{array}{lll}
F_{U}\left(\lambda_{0}\right) & \\
\ddots & & \\
& \ddots & \\
& & \ddots \\
& & F_{U}\left(\lambda_{2 M-1}\right)
\end{array}\right)
$$


converges to the spectral form of the covariance matrix of the $\left\{\underset{\sim}{u_{t}}\right\}$ process.

With these assumptions, if all the predetermined variables in the model were exogenous we could require that they permit general harmonic analysis, and the asymptotic properties of the Specfiml estimator could be derived on the same basis as Hannan \& Terrell [7]. But when the model contains lagged endogenous variables, we require the following further assumptions:

Assumption 1. The AR process involving the dependent variables is stable, i. e., the roots of

$$
\operatorname{det}\left\{B_{0}+B_{1} \theta+\cdots+B_{r} \theta^{r}\right\}=0
$$

lie outside the unit circle.

Assumption 2. The vector process $\left\{z_{t}\right\}$ of exogenous variables is stationary to the fourth order with absolutely continuous spectra and

$$
F_{Z}(\lambda)=\frac{1}{2 \pi} \sum_{-\infty}^{\infty} G_{Z}(\tau) \exp (-i \tau \lambda),
$$

where $F_{Z}(\lambda)$ is the spectral density matrix of the variables in $Z$, and

Also

$$
G_{Z}(\tau)=\lim _{T \rightarrow \infty} \frac{1}{T} \sum_{t=1}^{T} z_{t} z_{t+t}^{\prime}, \quad \tau=0,1, \ldots
$$

$$
\begin{aligned}
& \sum_{-\infty}^{\infty}|j|\left\|G_{Z}(\tau)\right\|<\infty, \\
& \operatorname{det} F_{Z}(\lambda)>0
\end{aligned}
$$

and $\{z$, $\}$ also has a fourth cumulant function that satisfics

$$
\sum_{\tau_{1}} \sum_{\tau_{2}} \sum_{\tau_{3}=-\infty}^{\infty}\left|K_{e, j, k, n}\left(0, \tau_{1}, \tau_{2}, \tau_{3}\right)\right|<\infty
$$

and

$$
G_{Z}(0) \text { is non-singular. }
$$

Assumption 3. $\lim T / M^{4} \longrightarrow 0$.

These extra conditions are probably unnecessarily strong, but we do not pursue here their relaxation.

Assumption 4. The model is identified. For the problem of identification we refer to Sargan [12] and Hannan [5], but basically what we mean by identified is the order of the AR process is known, the model has enough well behaved exogenous variables and the coefficients of which $A$ is comprised are subject to suitable set of zero and standardizing restrictions. We also require that conditions closely allied to the control theory concept of controllability be satisfied. A sufficient set of conditions allied to one set of such conditions given by Hannan 
[5] is that the matrix $\left(B_{r}: \Gamma\right)$ is of full rank $p$. In addition the usual econometric conditions for the identification of the structural form from the reduced form must be satisfied. For simplicity we assume that there are no identities in the model.

\section{ALTERNATIVE LIKELIHOOD FUNCTIONS}

Arguing as in Espasa [1] we can write the log likelihood function corresponding to model (1) as

$$
\begin{aligned}
\frac{L\left[\operatorname{Vec}\left(Y^{\prime}\right)\right]}{T}=L & =c-\frac{m}{2 T} \sum_{h} \log \operatorname{det} F_{U}\left(\lambda_{h}\right) \\
& -\frac{m}{2 T} \sum_{h} \operatorname{tr}\left\{F_{U}^{-1}\left(\lambda_{h}\right) A I_{X X}\left(\lambda_{h}\right) A^{\prime}\right\}+\log \left|\operatorname{det} B_{0}\right|,
\end{aligned}
$$

and using

$$
\hat{F}_{U}\left(\lambda_{h}\right)=I_{U}\left(\lambda_{h}\right)=A I_{X X}\left(\lambda_{h}\right) A^{\prime}
$$

the concentrated likelihood can be expressed as

$$
L_{c}=c_{1}+\log \left|\operatorname{det} B_{0}\right|-\frac{m}{2 T} \sum_{h} \log \operatorname{det} I_{U}\left(\lambda_{h}\right) .
$$

Now writing

$$
I I=-B_{0}^{-1} \Gamma^{\dagger}
$$

and

$$
P^{\prime}=\left(I^{\prime}: I\right)
$$

we can deduce first order conditions for the Specfiml estimators of the form

$$
\left[\sum_{h} \operatorname{vec} \hat{F}_{U}^{-1}\left(\lambda_{h}\right) \hat{A} I_{X Z^{\dagger}}\left(\lambda_{h}\right) P^{\prime}\right]_{u}=0 .
$$

Throughout this article a suffix $u$ attached to square brackets means that (i) in the case of a vec, only those elements which correspond to unconstrained coefficients in vec $A$ are to be considered, (ii) in the case of a square matrix, only those rows and columns which correspond to unconstrained elements of vec $A$ are to be retained; the other rows and columns are deleted.

If $r=0$, (i.e. there are no lagged endogenous variables), it was shown by Espasa [1] that this is a form from which the asymptotic distribution of the estimates can be derived, and that the asymptotic variance of the unconstrained elements of $\sqrt{T} \operatorname{vec}(\widehat{A}-A)$ can be estimated by

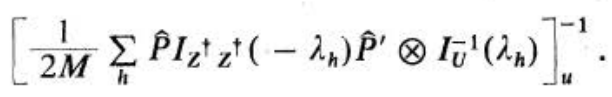

This leads to the suggested 3SLS estimator given by

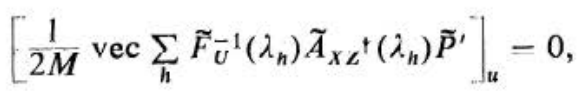


where $A$ is the Spectral 3SLS (Spec 3SLS) estimator based on consistent estimators of $F_{U}\left(\lambda_{h}\right)$ and $P$.

However, if $r \neq 0$

(a) (9) does not provide a suitable basis for discussing the asymptotic distribution of the estimators;

(b) (10) does not provide a consistent estimate of the asymptotic variance matrix;

(c) (11) does not give an $\tilde{A}$ asymptotically equivalent to $\hat{A}$.

To do this we use an alternative form of the likelihood function.

Writing

$$
B\left(\omega_{j}\right)=B_{0}+B_{1} \exp \left(-i \omega_{j}\right)+\cdots+B_{r} \exp \left(-i r \omega_{j}\right)
$$

we have that

$$
B\left(\omega_{j}\right) \bar{w}_{Y}\left(\omega_{j}\right)+\Gamma \bar{w}_{Z}\left(\omega_{j}\right)=\bar{w}_{U}\left(\omega_{j}\right)+0(1 / \sqrt{T})
$$

and the contribution of the residual term (see Hannan [4, (493)] is negligible.

Now assuming that the spectra of the errors are constant within bands, assuming continuity and using Assumption $3, L$ can be replaced by

$$
\begin{aligned}
L_{1}=c & -\frac{m}{2 T} \sum_{h} \log \operatorname{det} F_{U}\left(\lambda_{h}\right)-\frac{m}{2 T} \sum_{h} \operatorname{tr}\left\{F_{U}^{-1}\left(\lambda_{h}\right) \ddot{I}_{U}\left(\lambda_{h}\right)\right\} \\
& +\frac{m}{T} \sum_{h} \log \left|\operatorname{det} B\left(\lambda_{h}\right)\right|
\end{aligned}
$$

where

$$
\begin{aligned}
& \ddot{I}_{U}\left(\lambda_{h}\right)=A\left(\lambda_{h}\right) I_{\chi \chi}\left(\lambda_{h}\right) A^{*}\left(\lambda_{h}\right), \\
& \chi=(Y Z) \text { and } \\
& A\left(\lambda_{h}\right)=\left[B\left(\lambda_{h}\right): \Gamma\right]
\end{aligned}
$$

To reconcile $L_{1}$ with $L$ we need to justify the substitution of $\ddot{I}_{U}\left(\lambda_{h}\right)$ by $I_{U}\left(\lambda_{h}\right)$ and the different expression for the Jacobian contribution. The first point can be justified by using an argument similar to that employed in Hannan [4, (492495)], by putting our smoothed estimator for the spectra (the average periodogram, FFT in Hannan's terminology) in terms of the sample covariances and a lag window (Hannan [4, (275-76)]. And the second point is justified in the appendix of Espasa [2].

Concentrating as before, but now using

$$
\hat{F}_{U}\left(\lambda_{h}\right)=\ddot{I}_{U}\left(\lambda_{h}\right)
$$

we have

$$
L_{c}=c_{e}+\frac{1}{2 M} \sum_{h} \log \operatorname{det} B\left(\lambda_{h}\right)-\frac{1}{4 M} \sum_{h} \log \operatorname{det} \ddot{I}_{U}\left(\lambda_{h}\right) .
$$


Now, if we call

$$
\underline{\phi}=\left[\phi_{j}\right]=[\operatorname{Vec}(A)]_{u}
$$

we can differentiate $L_{e}$ with respect to any element of $\underline{\phi}$, and we get

$$
\begin{aligned}
& \frac{\partial L_{e}}{\partial \phi_{j}}=\frac{1}{2 M} \sum_{h} \operatorname{tr}\left[B^{\prime-1}\left(\lambda_{h}\right) \frac{\partial B^{\prime}\left(\lambda_{h}\right)}{\partial \phi_{j}}\right] \\
& -\frac{1}{2 M} \sum_{h} \operatorname{tr} \ddot{I}_{U}^{-1}\left(\lambda_{h}\right) A\left(\lambda_{h}\right) I_{\chi \chi}\left(\lambda_{h}\right)^{A^{*}\left(\lambda_{h}\right)} \frac{\partial \phi_{j}}{\partial \phi^{\prime}} .
\end{aligned}
$$

Now we write

$$
\Phi\left(\dot{\lambda}_{h}\right)=\left(I_{p}, e^{i \lambda_{h}} I_{p}, \ldots, e^{r i \lambda_{h}} I_{p}\right)
$$

and define

$$
P^{\prime}\left(\lambda_{h}\right)=\left[-\Gamma^{\prime} B^{\prime-1}\left(-\lambda_{h}\right) \Phi\left(\lambda_{h}\right): I\right] .
$$

Then noting that

$$
\frac{B^{\prime}\left(-\lambda_{h}\right)}{\partial \phi_{j}}=\Phi\left(\lambda_{h}\right) \frac{\partial B^{+^{\prime}}}{\partial \phi_{j}^{-j^{-}}},
$$

where

$$
B^{+}=\left(B_{0}, B_{1}, \ldots, B_{r}\right),
$$

we can after some manipulation write

$$
\frac{\partial L_{e}}{\partial \phi_{j}}=-\frac{1}{2 M} \sum_{h} \operatorname{tr}\left(\ddot{I}_{U}^{-1}\left(\lambda_{h}\right) \hat{A}\left(\lambda_{h}\right) I_{\chi Z}\left(\lambda_{h}\right) P^{\prime}\left(\lambda_{h}\right) \frac{\partial A^{\prime}}{\partial \phi_{j}}\right) .
$$

If the $A$ matrix has only the usual zero or standardizing restrictions the first order conditions can be written

$$
\left[\sum_{h} \operatorname{vec} \ddot{I}_{U}^{-1}\left(\lambda_{h}\right) \hat{A}\left(\lambda_{h}\right) I_{x Z}\left(\lambda_{h}\right) \hat{P}^{\prime}\left(\lambda_{h}\right)\right]_{u}=0 .
$$

Now (27) can be used to derive an asymptotic error variance matrix and to suggest a suitable 3SLS approximation. Indeed we can immediately write down a Spec 3SLS estimator of the form

$$
\left[\sum_{h} \operatorname{vec} \tilde{\tilde{I}}_{U}^{-1}\left(\lambda_{h}\right) A\left(\tilde{\lambda}_{h}\right) I_{z Z}\left(\lambda_{h}\right) \tilde{P}^{\prime}\left(\lambda_{h}\right)\right]_{u}=0
$$

or since

$$
A\left(\lambda_{h}\right) W_{\chi}^{*}\left(\lambda_{h}\right)=A W_{\chi}^{*}\left(\lambda_{h}\right)+R_{0},
$$

where $R_{0}$ is asymptotically negligible, as

$$
\left[\sum_{h} \operatorname{vec} \bar{I}_{U}^{-1}\left(\lambda_{h}\right) \tilde{A}_{x L}\left(\lambda_{h}\right) \tilde{P}^{\prime}\left(\lambda_{h}\right)\right]_{u}=0,
$$


where $\ddot{I}_{U}^{-1}\left(\lambda_{h}\right)$ and $\widetilde{P}\left(\lambda_{h}\right)$ are any consistent estimators of $F_{U}^{-1}\left(\lambda_{h}\right)$ and $P\left(\lambda_{h}\right)$, for instance, computed from the results of an instrumental variable estimator for $A$. If the $\tilde{A}$ we obtain are different from those used to compute $\tilde{\tilde{I}}_{U}^{-1}\left(\lambda_{h}\right)$ and $\widetilde{P}\left(\lambda_{h}\right)$ by non-trivial amounts, then it will pay to iterate by estimating $F_{U}^{-1}\left(\lambda_{h}\right)$ and $P\left(\lambda_{h}\right)$ using $\tilde{\tilde{A}}$.

\section{THE ASYMPTOTIC PROPERTIES OF THE SPECFIML ESTIMATORS}

To obtain the asymptotic distribution of $A$ we have that

$$
A I_{X Z}\left(\lambda_{h}\right)=I_{U Z}\left(\lambda_{h}\right)
$$

so that

$$
\begin{aligned}
& {\left[\frac{1}{2 M} \sum_{h} \hat{P}\left(\lambda_{h}\right) I_{Z X}\left(-\lambda_{h}\right) \otimes \ddot{I}_{U}^{-1}\left(\lambda_{h}\right)\right]_{u} \sqrt{T}(\hat{\phi}-\phi)} \\
& \quad=-\frac{\sqrt{m}}{\sqrt{2 M}}\left[\sum_{h}\left(\hat{P}\left(\lambda_{h}\right) \otimes \ddot{I}_{U}^{-1}\left(\lambda_{h}\right)\right) \operatorname{vec} I_{U Z}\left(\lambda_{h}\right)\right]_{u} .
\end{aligned}
$$

Note that using (13) and the results in Hannan [4, (chapter 7)], we have

$$
\begin{aligned}
\operatorname{pim}_{T \rightarrow \infty} & \frac{1}{2 M}\left[\sum_{h} \hat{P}\left(\lambda_{h}\right) I_{Z X}\left(-\lambda_{h}\right) \otimes \ddot{I}_{U}^{-1}\left(\lambda_{h}\right)\right]_{u} \\
= & \operatorname{pim}_{T \rightarrow \infty} \frac{1}{2 M}\left[\sum_{h} \hat{P}\left(\lambda_{h}\right) I_{Z Z}\left(-\lambda_{h}\right) \hat{P}^{*}\left(\lambda_{h}\right) \otimes \ddot{I}_{U}^{-1}\left(\lambda_{h}\right)\right]_{u} \\
\quad & {\left[\frac{1}{2 \pi} \int_{-\pi}^{\pi} P(\lambda) F_{Z Z}(-\lambda) P^{*}(\lambda) \otimes F_{U}^{-1}(\lambda) d \lambda\right]_{u} }
\end{aligned}
$$

and if the model is identified and in view of Assumption 2, following Hannan [4, (441)], this is non-singular since it is the integral of a positive definite Hermitian matrix.

Now under the present assumptions and arguing on the lines of Hannan [4, (492-495)], we can asymptotically replace the R. H. S. matrix of (30) by

$$
-\frac{\sqrt{m}}{\sqrt{2 M}}\left[\sum_{h} P\left(\lambda_{h}\right) \otimes F_{U}^{-1}\left(\lambda_{h}\right) \operatorname{vec} I_{U Z}\left(\lambda_{h}\right)\right]_{u},
$$

which, by Hannan's theorem (see Hannan [4, (442, 487-492)]), has an asymptotic multivariate normal distribution with variance matrix given by (31), so that $\sqrt{T}(\hat{\phi}-\phi)$ has an asymptotic multivariate normal distribution with zero mean and variance matrix given by the inverse of (31), and can be consistently estimated by

$$
\left[\frac{1}{2 M} \sum_{h} \hat{P}\left(\lambda_{h}\right) I_{Z Z}\left(-\lambda_{h}\right) \hat{P} *\left(\lambda_{h}\right) \otimes \ddot{I}_{U}^{-1}\left(\lambda_{h}\right)\right]_{u}^{-1} .
$$

When $r=0$, this agrees with the previous result derived in Hannan and Terrell [7] and in Espasa [1] where it is shown that there is no loss in efficiency when 
assuming a general stationary structure (G.S.S.) for the errors if they in fact follow a finite autoregressive process.

Note that (33) also gives an estimate of the asymptotic variance matrix of the errors in the estimators obtained by maximizing (5) and that leads us to the problems of computation that we treat in the next section. But if the spectral estimators were to be computed from the first order conditions, it would be simpler to use (9) rather than (27) and the difference is asymptotically negligible provided that Assumption 1 is satisfied.

Note that the estimates obtained by maximizing $L_{e}$ have the same limitations as those obtained by maximizing $L$, i. e., we must have $m>p(r+1)+k$, (see Espasa [1]). Moreover, if we look at the similar conditions for the Spec 3SLS defined by (29), we require also that $\tilde{I}_{U}^{-1}\left(\lambda_{h}\right)$ is non-singular, which requires that $p<m$. We obviously require that the estimate of $A, \widetilde{A}$, on which the estimate of $\widetilde{P}\left(\lambda_{h}\right)$ is based should lead to non-singular $\widetilde{B}\left(\lambda_{h}\right)$ for all $h$ as required by Assumption 1, and also that the matrix

$$
\left[\sum_{\lambda_{h}} \tilde{P}\left(\lambda_{h}\right) I_{Z X}\left(-\lambda_{h}\right) \otimes \tilde{I}_{U}^{-1}\left(\lambda_{h}\right)\right]_{u}
$$

should be non-singular. These conditions will be satisfied with probability one as $T \rightarrow \infty$, if the model is correctly specificd.

\section{THE COMPUTATION OF THE SPECFIML ESTIMATORS}

In this section we explain the procedure for computing the spectral estimators of a simultaneous equations model, which is the basis for the Fortran program, Specfiml, written by the first author for the computations shown in the appendix. The approach followed in the construction of the program is closely related to the one used in Hendry $[8,9]$ and consists of minimizing minus the likelihood function by means of a Powell [11] subroutine that calculates the minimum of a function without using derivatives. The standard errors of the estimators are obtained by a numerical calculation of the Hessian. Besides the estimators of the structural coefficients, their standard errors and $t$ values, the Specfiml program also gives the cospectra, quadrature-spectra and coherency matrices at different frequency bands of the estimated disturbances and this output can also be obtained with the initial values and at the different stages of the iteration process, if required.

It was proved in the appendix of [2] that the estimates resulting from the maximization of $L_{e}$ defined in (19) are asymptotically equivalent to those obtained from the maximization of $L_{c}$ defined in (7). Since it is computationally simpler to obtain $L_{c}$ than $L_{e}$, the criterion function that we choose to minimize in the Specfiml program is

$$
f=-\log \operatorname{det} B_{0}+\frac{1}{T} \sum_{h=0}^{M} \delta_{h} m_{h} \log \operatorname{det} A I_{X X}\left(\lambda_{h}\right) A^{\prime}
$$


with $B_{0}, A$ and $X$ defined as in Section 2 and $\delta_{h}=1$ except that $\delta_{0}=\delta_{M}=\frac{1}{2}$. Obviously $f$ is the same as the criterion function used by Espasa in [1] in the special case where there are no lagged endogenous variables. Also since the Hessians of $L_{c}$ and $L_{c}$ are asymptotically equivalent, the computation procedure in the Specfiml program gives, for large $T$ and $M$, a consistent estimate of the asymptotic variance matrices, whether the model includes lagged values of the endogenous variables or not.

The program uses as input the values of the elements of the matrices $I_{X X}\left(\lambda_{h}\right)$, but minor modifications will allow it to use $I_{X X}\left(\omega_{j}\right)$ or even $W_{X}\left(\omega_{j}\right)$ as inputs. $I_{X X}\left(\lambda_{h}\right)$ was produced by a separate program using a Fast Fourier subroutinc. It was decided not to combine the two programs and use $X$ as input, because in small samples the selection of the band-width may be crucial and some experimentation with different sets of $m_{h}$ 's values may be worthwhile. It would be a waste of time to recompute $W_{X}\left(\omega_{j}\right)$ at each trial.

The program calculates $\log \operatorname{det} I_{U}\left(\lambda_{h}\right)=\log \operatorname{det} A I_{X X}\left(\lambda_{h}\right) A^{\prime}$ by means of a subroutine designed to invert Hermitian positive definite matrices using complex numbers. But since the determinant of an Hermitian matrix is real, the computations of $\log \operatorname{det} I_{U}\left(\lambda_{h}\right)$ could alternatively be based on real matrices. In fact, if we split $I_{U}\left(\lambda_{h}\right)$ into its real and imaginary parts so that

$$
I_{U}\left(\lambda_{h}\right)=R_{U}\left(\lambda_{h}\right)-i C\left(\lambda_{h}\right),
$$

an alternative form of the criterion function involving real matrices only is

$$
\begin{aligned}
f= & -\log \operatorname{det} B_{0}+\frac{1}{T} \sum_{h=0}^{M} \delta_{h} m_{h}\left\{\log \operatorname{det} R_{U}\left(\lambda_{h}\right)\right. \\
& \left.+\log \operatorname{det}\left[R_{U}\left(\lambda_{h}\right)+C_{U}\left(\lambda_{h}\right) R_{U}^{-1}\left(\lambda_{h}\right) C_{U}\left(\lambda_{h}\right)\right]\right\} .
\end{aligned}
$$

Finally the concentrated likelihood could be put in terms of the observation matrix in a real formulation by noting that either,

$$
\begin{aligned}
& \log \operatorname{det}\left[A I_{X X}\left(\lambda_{h}\right) A^{\prime}\right] \\
& =\log \operatorname{det}\left\{(A A)\left(\begin{array}{cc}
X^{\prime} & 0 \\
0 & X^{\prime}
\end{array}\right)\left(\begin{array}{cc}
Q_{1}^{\prime}\left(\lambda_{h}\right) & 0 \\
0 & Q_{2}^{\prime}\left(\lambda_{h}\right)
\end{array}\right)\left(\begin{array}{cc}
Q_{1}\left(\lambda_{h h}\right) & 0 \\
0 & Q_{2}\left(\lambda_{h}\right)
\end{array}\right)\left(\begin{array}{cc}
X & 0 \\
0 & X
\end{array}\right)\left(\begin{array}{l}
A^{\prime} \\
A^{\prime}
\end{array}\right)\right\}, \\
& \text { if } h=0 \text { and } M,
\end{aligned}
$$

or,

$$
=\log \operatorname{det}\left(\begin{array}{cc}
A X^{\prime} \psi_{1}\left(\lambda_{h}\right) X A^{\prime} & A X^{\prime} \psi_{2} X A^{\prime} \\
-A X^{\prime} \psi_{2}\left(\lambda_{h}\right) X A^{\prime} & A X^{\prime} \psi_{1} X A^{\prime}
\end{array}\right), \quad \text { if } h=1, \ldots, M-1,
$$

where $Q_{1}\left(\lambda_{h}\right)$ and $Q_{2}\left(\lambda_{h}\right)$ are defined from

$$
\frac{Q\left(\lambda_{h}\right)}{\sqrt{2 \pi m_{h}}}=Q_{1}\left(\lambda_{h}\right)+i Q_{2}\left(\lambda_{h}\right)
$$


and

$$
\begin{aligned}
& \Psi_{1}\left(\lambda_{h}\right)=Q_{1}^{\prime}\left(\lambda_{h}\right) Q_{1}\left(\lambda_{h}\right)+Q_{2}^{\prime}\left(\lambda_{h}\right) Q_{2}\left(\lambda_{h}\right) \text { and } \\
& \Psi_{2}\left(\lambda_{h}\right)=Q_{2}^{\prime}\left(\lambda_{h}\right) Q_{1}\left(\lambda_{h}\right)-Q_{1}^{\prime}\left(\lambda_{h}\right) Q_{2}\left(\lambda_{h}\right) .
\end{aligned}
$$

\section{THE ASYMPTOTIC VARIANCE MATRIX OF THE STRUCTURAL ESTIMATORS WHEN THE ERRORS FOLLOW AN AR PROCESS}

It is interesting to compare the asymptotic properties of the Specfiml estimators with the asymptotic properties of the Maximum Likelihood estimators when the errors follow a vector moving average process. ${ }^{3}$ There is no difficulty in proving that the results obtained in Hannan and Nicholls [6] can be generalized here for the simultaneous model.

Similar conclusions are obtained when comparing the Specfiml estimators with the maximum likelihood (ML) estimators if the errors follow an AR process. In order to prove this we devote this section to obtaining asymptotic variance matrix of the ML estimators in the AR case.

The model considered here is the one set up in the previous sections, but now the errors are generated by a vector autoregressive model of the form

$$
{\underset{\sim}{t}}_{t}-R_{1}{\underset{\sim}{t-1}}_{t}, \ldots, R_{r} u_{t-\tilde{r}}={\underset{q}{t}}_{t} .
$$

We find it convenient to work with the reduced form, which we write

$$
y_{t}-\Pi_{z}^{z_{t}^{\dagger}}={\underset{\sim}{v}}_{t},
$$

where $\Pi$ and $z_{t}^{\dagger}$ are as defined in (3) and (8) respectively and

$$
\begin{aligned}
& \underset{\sim}{v}-S_{1} v_{t-1}-\cdots-S_{r} v_{t-\tilde{r}}=\xi_{t}^{0} \\
& \underset{t}{v_{t}}=B_{0}^{-1} \underline{u}_{t}, \quad S_{k}=B_{0}^{-1} R_{k} B_{0}, \\
& \dot{\varepsilon}_{t}^{0}=B^{-1} \xi_{t} .
\end{aligned}
$$

Then we have

$$
\operatorname{Var}\left(\varepsilon_{t}\right)=G \text { and } \operatorname{Var}\left(\varepsilon_{t}^{0}\right)=G^{0} .
$$

To find the AVM of $\sqrt{T}(\hat{\hat{A}}-A)$, where $\hat{A}$ is the time domain autoregressive ML estimator, we consider the first and second derivatives of the likelihood function. We can write the concentrated likelihood function as

$$
L_{r}=-\frac{1}{2} T \log \operatorname{det}\left(E^{0^{\prime}} E^{0}\right),
$$

where $E^{0 \prime}$ is a matrix formed with the column vectors $\varepsilon_{t}^{0}$ and we assume that the

${ }^{3}$ See Nicholls [10] for the estimation of a simultaneous model with a vector moving average error process. 
$\underset{\sim}{\varepsilon_{t}^{0}}$ have been expressed as functions of some set of parameters $\underset{\sim}{\phi}$, eliminating the $S_{k}$ by using the equations

$$
\sum_{k=0}^{\tilde{r}} V_{j}^{\prime} V_{k} S_{k}^{\prime}=0, \quad j=1, \ldots, \tilde{r},
$$

where $V_{k}$ is the matrix whose rows are $\underset{t-k}{v}, t=1, \ldots, T$ and $S_{0}=-I$. Then $\underset{\sim}{\underset{\delta}{q}} 0$ is determined from $v_{t-s}$ using (35) and (37) and then is expressed as a function of $A$ from (34) and (8). We assume that $A$ depends upon the $N$ parameters of the vector $\phi$.

For the Hessian we have

$$
\begin{aligned}
\frac{\partial^{2} L_{r}}{\partial \phi_{n} \partial \phi_{q}}= & -T \operatorname{tr}\left[\frac{\partial}{\partial \phi_{q}}\left(\hat{G}^{0-1}\right)\left(E^{0^{\prime}} \frac{\partial E^{0}}{\partial \phi_{n}}\right)\right] \\
& -T \operatorname{tr}\left[\hat{G}^{0-1}\left(E^{0 \prime} \frac{\partial^{2} E^{0}}{\partial \phi_{n} \partial \phi_{q}}\right)\right]-T \operatorname{tr}\left[\hat{G}^{0-1}\left(\frac{\partial E^{0 \prime}}{\partial \phi_{q}}\right)\left(\frac{\partial E^{0}}{\partial \phi_{n}}\right)\right] .
\end{aligned}
$$

Note that the first two terms are $O(\sqrt{T})$ since $E^{0 \prime}$ in each occurs multiplied by $Z_{s}^{\prime}$ when explicit expression for $\partial E^{0} / \partial \phi_{n}$ and $\partial^{2} E^{0} / \partial \phi_{n} \partial \phi_{q}$ are substituted, and so can be neglected compared with the last term which is $0(T)$. In this term we must take account of the dependence in $\frac{\partial E^{0}}{\partial \phi_{q}}$ of $S_{s}$ on $\phi_{q}$.

Differentiating (37) we have

$$
\frac{\partial \Pi}{\partial \phi_{n}} \sum_{k=0}^{\tilde{r}}\left(Z_{j}^{\dagger \prime} V_{k}\right) S_{k}^{\prime}+\sum_{k=0}^{\tilde{r}}\left(V_{j}^{\prime} Z_{k}^{\dagger}\right)\left(\frac{\partial \Pi}{\partial \phi_{n}}\right)^{\prime} S_{k}^{\prime}-\sum_{k=1}^{\tilde{r}}\left(V_{j}^{\prime} V_{k}\right) \frac{\partial S_{k}^{\prime}}{\partial \phi_{n}}=0 .
$$

Now $\sum_{k=0}^{\tilde{r}}\left(Z_{j}^{\dagger} V_{k}\right) S_{k}^{\prime}=Z_{j}^{\dagger} E^{0}=0(\sqrt{T})$,

and so the first term is negligible. So now write

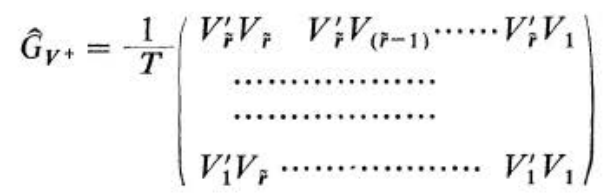

and

$$
K_{n}=\left(\begin{array}{c}
\sum_{k=0}^{\tilde{r}} V_{\tilde{r}}^{\prime} Z_{k}^{\dagger}\left(\frac{\partial \Pi}{\partial \phi_{n}}\right)^{\prime} S_{k}^{\prime} \\
\cdots \cdots \cdots \cdots \cdots \cdots \cdots \\
\cdots \cdots \cdots \cdots \cdots \cdots \cdots \cdots \\
\sum_{k=0}^{\tilde{r}} V_{1}^{\prime} Z_{k}^{\dagger}\left(\frac{\partial \Pi}{\partial \phi_{n}}\right)^{\prime} S_{k}^{\prime}
\end{array}\right) .
$$

Then we have 


$$
\left(\begin{array}{c}
\frac{\partial S_{\dot{r}}^{\prime}}{\partial \phi_{n}} \\
\cdots \cdots \\
\cdots \cdots \\
\frac{\partial S_{1}^{\prime}}{\partial \phi_{n}}
\end{array}\right)=\hat{G}_{V}^{-1}+K_{n}
$$

and

$$
\frac{d E^{0^{\prime}}}{d \phi_{q}}=\frac{\partial E^{0 \prime}}{\partial \phi_{q}}-K_{q}^{\prime} \hat{G}_{V^{+}}^{-1}\left(\begin{array}{c}
V_{\dot{p}}^{\prime} \\
\vdots \\
V_{1}^{\prime}
\end{array}\right)
$$

so that

$$
\begin{aligned}
& \frac{1}{T} \frac{\partial^{2} L}{\partial \phi_{n} \partial \phi_{q}}=-\operatorname{tr}\left[\left(E^{0^{\prime} E}\right)^{-1}\left(\frac{\partial E}{\partial \phi_{q}}\right)^{\prime}\left(\frac{\partial E^{0}}{\partial \phi_{n}}\right)\right] \\
& +\operatorname{tr}\left\{\left(E^{0^{\prime}} E^{0}\right)^{-1} K_{q}^{\prime} \hat{G}_{V^{+}}^{-1}\left(\begin{array}{c}
V_{\tilde{r}}^{\prime} \\
\vdots \\
V_{1}^{\prime}
\end{array}\right) \sum_{s=0}^{\tilde{r}} Z_{s}^{\dagger}\left(\frac{\partial \Pi}{\partial \phi_{n}}\right)^{\prime} S_{s}\right\} \\
& =-\operatorname{tr}\left\{\left(\frac{E^{0^{\prime}} E^{0}}{T}\right)^{-1} \sum_{k} \sum_{s}\left[S_{k}\left(\frac{\partial \Pi}{\partial \phi_{q}}\right)\left(\frac{Z_{k}^{\prime} Z_{s}^{\dagger}}{T}\right)\left(\frac{\partial \Pi}{\partial \phi_{n}}\right)^{\prime} S_{s}^{\prime}\right]\right\} \\
& +\operatorname{tr}\left\{\left(\frac{E^{0 \prime} E^{0}}{T}\right)^{-1} K_{q}^{\prime} \hat{G}_{V^{\prime}}^{-1} K_{n}\right\} \text {. }
\end{aligned}
$$

Now write

$$
\Xi_{\phi}=-\left(\operatorname{plim}\left(\frac{\partial^{2} L_{r}}{\partial \phi_{n} \partial \phi_{q}} / T\right)\right)=\left[\xi_{\phi}^{n q}\right]=\Omega_{\phi}^{-1},
$$

and

$$
\begin{aligned}
& G_{V V}(\tau)=\operatorname{plim}\left(\frac{V_{k+\tau}^{\prime} V_{k}}{T}\right) \\
& G_{V Z^{\dagger}}(\tau)=\operatorname{plim}\left(\frac{V_{k+\tau}^{\prime} Z_{k}^{\dagger}}{T}\right) \\
& G_{Z^{\dagger} Z^{\dagger}}(\tau)=\operatorname{plim}\left(\frac{Z_{k+\tau}^{\prime \prime} Z_{k}^{\dagger}}{T}\right) .
\end{aligned}
$$

Then we have

$$
\begin{aligned}
& \xi_{\phi}^{n q}=\operatorname{tr}\left\{G^{0-1} \sum_{k=0}^{\tilde{r}} \sum_{s=0}^{\tilde{f}}\left[S_{k}\left(\frac{\partial \Pi}{\partial \phi_{q}}\right) G_{Z^{\dagger} z^{\dagger}}(k-s)\left(\frac{\partial \Pi}{\partial \phi_{n}}\right)^{\prime} S_{s}^{\prime}\right]\right\} \\
& -\operatorname{tr}\left\{G^{0-1} \tilde{K}_{q}^{\prime} G_{V^{+}(\tilde{r})}^{-1} \tilde{K}_{n}\right\},
\end{aligned}
$$

where 


$$
\begin{aligned}
& \tilde{K}_{q}=\operatorname{plim}_{T \rightarrow \infty} K_{q}=\left(\sum_{k} G_{V Z^{\dagger}}(\tilde{r}-k)\left(\frac{\partial \Pi}{\partial \phi_{q}}\right)^{\prime} S_{k}^{\prime}\right. \\
& \sum_{k} G_{V Z^{\dagger}}(1-k)\left(\frac{\partial \Pi}{\partial \phi_{q}}\right)^{\prime} S_{k}^{\prime} \\
& \tilde{G}_{V^{+}(\tilde{r})}^{-1}=\operatorname{plim}_{T \rightarrow \infty} \hat{G}_{V^{+}}^{-1}=\left(\begin{array}{cccc}
G_{V V}(0) & G_{V V}(1) & \cdots & G_{V V}(r-1) \\
G_{V V}(-1) & \ddots & \vdots \\
\vdots & & \ddots & \vdots \\
G_{V V}(-\tilde{r}+1) & \cdots \cdots G_{V V}(0)
\end{array}\right)^{-1}
\end{aligned}
$$

Now we consider the case where the order of the AR process is $r^{+}$, but we mistakenly assume in estimation that the order is $\tilde{r}>r^{+}$, and in particular we consider the limit of $\xi_{\phi}^{n q}$ as $\tilde{r} \rightarrow \infty$ with $S_{s}, s \leq r^{+}$remaining constant and $S_{s}=0$, $s>r^{+}$.

First we note that increasing $\tilde{r}$ in general increases the AVM.

Since the first term in $\xi_{\phi}^{n q}$ is independent of $\tilde{r}$, given $S_{s}=0, s>r^{+}$, we consider only the second term and if we have that minus the second term is increasing in $\tilde{r}$, then the AVM $\left(\Omega_{\phi}\right)$ will also be increasing.

More formally write $\Omega_{\phi}^{\tilde{r}}$ for the AVM corresponding to autoregressive order $\tilde{r}$, then if and only if

$$
\Omega_{\phi}^{\tilde{r}+1} \geq \Omega_{\phi}^{\tilde{r}}
$$

we have

$$
\left(\Omega_{\phi}^{\tilde{r}+1}\right)^{-1} \leq\left(\Omega_{\phi}^{\tilde{r}}\right)^{-1}
$$

and then for an arbitrary constant vector $\underset{\sim}{\alpha}$ we have

$$
\alpha^{\prime}\left[\left(\Omega_{\phi}^{\tilde{r}+1}\right)^{-1}\right] \alpha \leq \alpha^{\prime}\left[\left(\Omega_{\phi}^{\tilde{r}}\right)^{-1}\right] \alpha .
$$

Now write

$$
\Delta=\sum_{p=1}^{N} \alpha_{p} \frac{\partial \Pi}{\partial \phi_{p}}
$$

and

$$
\Delta_{\tilde{r}}=\left[\begin{array}{c}
\sum_{k=0}^{\tilde{r}} G_{V Z^{\dagger}}(\tilde{r}-k) \Delta^{\prime} S_{k}^{\prime} \\
\sum_{k=0}^{\tilde{r}} G_{V Z^{\dagger}}(\tilde{r}-k-1) \Delta^{\prime} S_{k}^{\prime} \\
\ldots \ldots \ldots \ldots \ldots \ldots \ldots \ldots \ldots \ldots \ldots \\
\cdots \ldots \ldots \ldots \ldots \ldots \ldots \ldots \\
\sum_{k=0}^{\tilde{r}} G_{V Z}{ }^{\dagger}(1-k) \Delta^{\prime} S_{k}^{\prime}
\end{array}\right]
$$

Then 


$$
\operatorname{tr}\left\{G^{0-1} \sum_{p} \sum_{q} \alpha_{p} \widetilde{K}_{p}^{\prime} G_{V^{+}(\tilde{r})}^{-1} \widetilde{K}_{q} \alpha_{q}\right\}=\operatorname{tr}\left(G^{0-1} \Delta_{\tilde{r}}^{\prime} G_{V^{+}(\tilde{r})}^{-1} \Delta_{\tilde{r}}\right),
$$

and if this increases with $\tilde{r}$, then so does the AVM, but now it is clear that

$$
\Delta_{(\tilde{r}+1)}^{\prime} G_{V}^{-1}(\tilde{r}+1) \Delta_{(\tilde{r}+1)} \geq \Delta_{\tilde{r}}^{\prime} G_{V^{+}(\tilde{r})}^{-1} \Delta_{\tilde{r}}
$$

by using the partitioned inverse of $G_{V^{+(\tilde{r}+1)}}$. The condition for strict inequality is rather complex, so that we now concentrate on the limit as $r \rightarrow \infty$.

The first term in (39) is relatively simple to express in spectral terms as

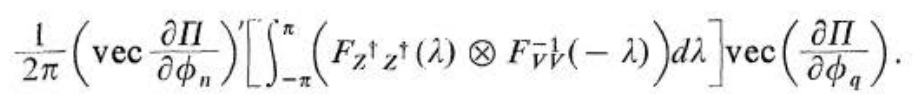

where $F_{Z}^{\dagger} Z^{\dagger}(\lambda)$ is the matrix spectral density corresponding to $G_{Z}^{\dagger} Z^{\dagger}(\tau)$, and writing $F_{V V}(\lambda)$ as the spectral density matrix corresponding to $G_{V V}(\tau)$.

This, of course, equals the usual formula for the AVM when $r=0$ (i.e. no lagged endogenous variables).

Now, for the second term of (39), it can be shown that as $\tilde{r} \rightarrow \infty$ it can be written as

$$
\left(\operatorname{vec}\left(\frac{\partial \Pi}{\partial \phi_{q}}\right)\right)^{\prime} \int_{-\pi}^{\pi}\left(F_{Z^{\dagger} V}(\lambda) F_{V V}^{-1}(\lambda) F_{V Z}{ }^{\dagger}(\lambda)\right) \otimes F_{V V}(-\lambda) d \lambda\left(\operatorname{vec}\left(\frac{\partial \Pi}{\partial \phi_{n}}\right)\right) .
$$

Thus compactly we can write

$$
\begin{aligned}
\xi_{\phi}^{n q}=\left(\operatorname{vec}\left(\frac{\partial \Pi}{\partial \phi_{n}}\right)\right)^{\prime} \int_{-\pi}^{\pi}\left[F_{Z^{\dagger} Z^{\dagger}}(\lambda)-F_{Z^{\dagger} V}(\lambda) F_{V V}^{-1}(\lambda) F_{V Z}{ }^{\dagger}(\lambda)\right] \\
\\
\otimes F_{V V}^{-1}(-\lambda) d \lambda\left(\operatorname{vec}\left(\frac{\partial \Pi}{\partial \phi_{q}}\right)\right)
\end{aligned}
$$

and that

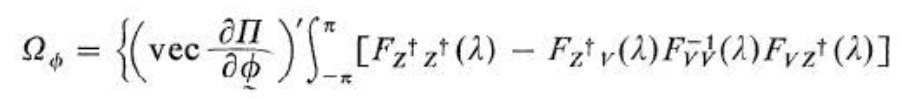

$$
\left.\otimes F_{V V}^{-1}(-\lambda) d \lambda \operatorname{vec}\left(\frac{\partial \Pi}{\partial \underline{\phi}}\right)\right\}^{-1},
$$

where vec $\frac{\partial \Pi}{\partial \phi}$ is the $\left(p k^{\dagger} \times n\right)$ matrix whose $j^{t h}$ row is $\frac{\partial}{\partial \phi_{j}}(\operatorname{vec} \Pi)^{\prime}$, and $k^{\dagger}$, the number of predetermined variables, equals $(p r+k)$.

Now $F_{Z V}(\lambda)=0$, and the elements of $F_{Z}^{* \dagger} z^{\dagger}(\lambda)-F_{V Z}{ }^{\dagger}(\lambda) F_{V V}^{-1}(\lambda) F_{V Z}{ }^{\dagger}(\lambda)$ only differ from $F_{Z}^{\dagger} z^{\dagger}(\lambda)$ when both row and column correspond to lagged endogenous variables.

Making use of the reduced form equation (34) it follows that the whole asymptotic variance matrix can now be written

$$
\begin{array}{r}
\Omega_{\phi}=\left\{\left(\operatorname{vec} \frac{\partial \Pi}{\partial \underline{\phi}}\right)^{\prime}\left[\int_{-\pi}^{\pi}\left(\left[F_{Z}^{\dagger}{ }_{Z}(\lambda) F_{Z Z}^{-1}(\lambda) F_{Z Z}^{\dagger}(\lambda)\right] \otimes F_{V V}^{-1}(-\lambda)\right) d \lambda\right]\right. \\
\left.\left(\operatorname{vec} \frac{\partial \Pi}{\partial \phi}\right)\right\}^{-1} .
\end{array}
$$


This is perfectly general for the case when the reduced form matrix $\Pi$ is a general function of a set of parameters $\phi$. Now consider the standard simultaneous equation case, where

$$
\frac{\partial \Pi}{\partial \underline{\phi}}=-B_{0}^{-1}\left(\frac{\partial A}{\partial \phi}\right) P
$$

Then

$$
\Omega_{\phi}=\left\{\left(\operatorname{vec} \frac{\partial A}{\partial \underline{\phi}}\right)^{\prime} \int_{-\pi}^{\pi}\left[F_{X Z}(\lambda) F_{Z Z}^{-1}(\lambda) F_{Z X}(\lambda) \otimes F_{v U}^{-1}(-\lambda)\right] d \lambda\left(\operatorname{vec} \frac{\partial A}{\partial \underline{\phi}}\right)\right\}^{-1},
$$

where $\left(\operatorname{vec} \frac{\partial A}{\partial \phi}\right)$ is $\left(p^{2}(r+1+k) \times n\right)$ matrix defined in an analogous way as vec $\frac{\partial \Pi}{\partial \phi}$ above.

If the restrictions are linear, or zero-one restrictions, then we can simply replace $\operatorname{vec}\left(\frac{\partial A}{\partial \phi}\right)$ by $S$, which, in the latter case, is a selection matrix.

Finally, noting that

$$
F_{X Z}(\lambda) F_{Z Z}^{-1}(\lambda) F_{Z X}(\lambda)=P(-\lambda) F_{Z Z}(\lambda) P^{*}(-\lambda),
$$

where $P(\lambda)$ was defined in (23), it is clear that the resulting AVM is the one derived in Section 4 for the Specfiml estimators.

\section{GENERAL COMMENT}

The last section makes it clear how much we depend, when we generalize the stochastic process generating the errors on our structural equations, on the assumption that there is a set of exogenous variables, completely incoherent with the errors on the equations which we are estimating. This is worrying, because there is a serious problem of inadequate sample size in the estimation of these spectral estimators, so that only relatively small models can be estimated, as discussed in Espasa [1]. In highly aggregate models, the aggregative variables which may be treated as exogenous should usually more properly be considered endogenous, and the resulting inconsistencies may be serious. Ignoring the inadequate sample size problem, the set of variables, which it may be really appropriate to consider exogenous, may behave like a set of trends or dummy variables which lead to estimates of the model coefficients with large standard errors. Of course, the same will be true if general ARMA models are used in the time domain with high order moving average and/or autoregressive equations. But in this context it seems a natural procedure to reduce the order of the stochastic processes by using the usual type of significance test, and in doing so, we end up with an acceptable explanation of the data, in which the error generation stochastic processes have been simplified, in effect allocating as much as possible of the explanation of the behavior of the observed data to the structural equation 
model. The econometrician would agree that this may be a rather arbitrary procedure, and would judge the value of the result largely on a priori judgements of the economic acceptability of the model. A comparison with the Specfiml estimates of the model would certainly throw light on the limitations of the ARMA pretesting estimates.

In the case of variables which are generated by a stochastic model outside the economic model which is being estimated, a time domain approach may be advantageous, if it is assumed that the errors on the two models are incoherent, and that there are rather long lags in the effect of economic variables on the variables generated by the outside model. In this case there will be no harm in the time domain in treating the outside variables as exogenous, provided the internal errors stochastic model is correctly specified. Of course, if the combined model is sufficiently stable and the outside lags are sufficiently long, the treatment of the outside variables as exogenous in spectral methods would lead to only small inconsistencies. An alternative treatment when using spectral methods would be to develop methods of estimating an incomplete set of equations. Thus equations of the form (29) can be used, dropping the requirement that there are as many equations as current endogenous variables, and using for $Z$ any set of suitable instrumental variables (i. e., variables which are exogenous from the point of view of the combined model generating both inside and outside variables).

$P^{\prime}\left(\lambda_{h}\right)$ can be replaced by $I_{Z Z}\left(\lambda_{h}\right)^{-1} I_{Z X}\left(\lambda_{h}\right)$, and the asymptotic error variance matrix estimated as

$$
\left[\sum_{h} \tilde{\ddot{I}}_{U}\left(\lambda_{h}\right) \otimes I_{X Z}\left(-\lambda_{h}\right) I_{Z Z}\left(-\lambda_{h}\right)^{-1} I_{Z X}\left(-\lambda_{h}\right)\right]_{u}^{-1} .
$$

As a special case we can consider similarly the estimation of a single equation.

Banco de España, Madrid, Spain

London School of Economics, United Kingdom

\section{APPENDIX}

The Application of the Spectral Estimation Method

We first apply the Specfiml method to the following artificial model:

$$
\begin{aligned}
& y_{1 t}=a_{1} z_{1 t}+a_{2} z_{2 t}+a_{3} z_{3 t}+u_{1 t} \\
& y_{2 t}=b_{1} y_{1 t}+b_{2} z_{2 t}+b_{3} z_{3 t}+u_{2 t} .
\end{aligned}
$$

The data ${ }^{1}$ have been generated with

$$
\begin{aligned}
& b_{1}=0.5, a_{1}=a_{2}=a_{3}=b_{2}=b_{3}=-1.0 \\
& z_{1 t}=0.8 z_{1(t-1)}+u_{3 t}
\end{aligned}
$$

1 We are grateful to D. Hendry for supplying the data. 


$$
\begin{aligned}
& z_{2 t}=0.5 z_{2(t-1)}+u_{4 t} \\
& z_{3 t}=u_{5 t}
\end{aligned}
$$

and

$$
\begin{array}{lll}
u_{1} \text { is i.i.d. } N\left(0, \sigma_{1}^{2}\right) & \sigma_{1}^{2}=0.09 \\
u_{2} \text { is } " & N\left(0, \sigma_{2}^{2}\right) & \sigma_{2}^{2}=\sigma_{3}^{2}=1.0 \\
u_{3} \text { is } " & N\left(0, \sigma_{3}^{2}\right) & \sigma_{3}^{2}=0.36 \\
u_{4} \text { is } " & N\left(0, \sigma_{4}^{2}\right) & \sigma_{4}^{2}=0.75 \\
u_{5} \text { is } " N\left(0, \sigma_{5}^{2}\right) &
\end{array}
$$

and $u_{1}, u_{2}, u_{3}, u_{4}$ and $u_{5}$ are independently distributed.

With 58 observations we have estimated the model by Ordinary Least Squares (OLS), Full Information Maximum Likelihood (FIML) and Specfiml. In the last case we have distinguished eight frequency bands between 0 and $2 \pi$ and we have done the estimation using all the frequency bands and also for each of the bands separately. The results are in Table 1 . Note that the model is recursive and all the estimation methods are efficient.

From these results we have that the Specfiml method has produced very sensible estimates of the parameters and of their standard errors. Also looking at the confidence intervals for the estimated spectra of the residuals from Specfiml, the white noise hypothesis is not rejected. It is interesting to note that, applying the Specfiml method to each of the five different frequency bands, we see that the results for the frequency band around zero are the ones which dominate the estimation results for all bands. This is not surprising given the way in which we have generated the system. Also note that, except for the band around zero, the DH's (see table 1) in the single frequency estimation are smaller than the corresponding ones in the over-all frequency estimation, only at the expense of a high coherency.

We now apply the Specfiml method to a four-equation wages-earnings-prices inflation model. This model is a slight variant of one model derived and discussed in Espasa [3]. The data, referring to U. K., is quarterly and the sample period goes from 1950(2)-1971(1), i.e. 84 observations. We have distinguished four frequency bands centered around $-\pi / 2,0, \pi / 2$ and $\pi$. Even with $2 M=4$ the number of periodogram ordinates $\left(m_{h}\right)$, for the frequency band around $\pi / 2$ is less than the number of variables (NV) in the model. In Espasa [1] it is shown that under certain general conditions $m_{h}$ must be equal to or greater than NV. One of those conditions is that each exogenous variable appears at least in two equations and this is not the case for the present model. Nevertheless, the results that we have obtained do not suggest a badly behaving likelihood function.

The results are in Table 2 and comparing with the ones reported in Espasa [3] we have that the Specfiml estimates agree quite well with the FIML estimates. 


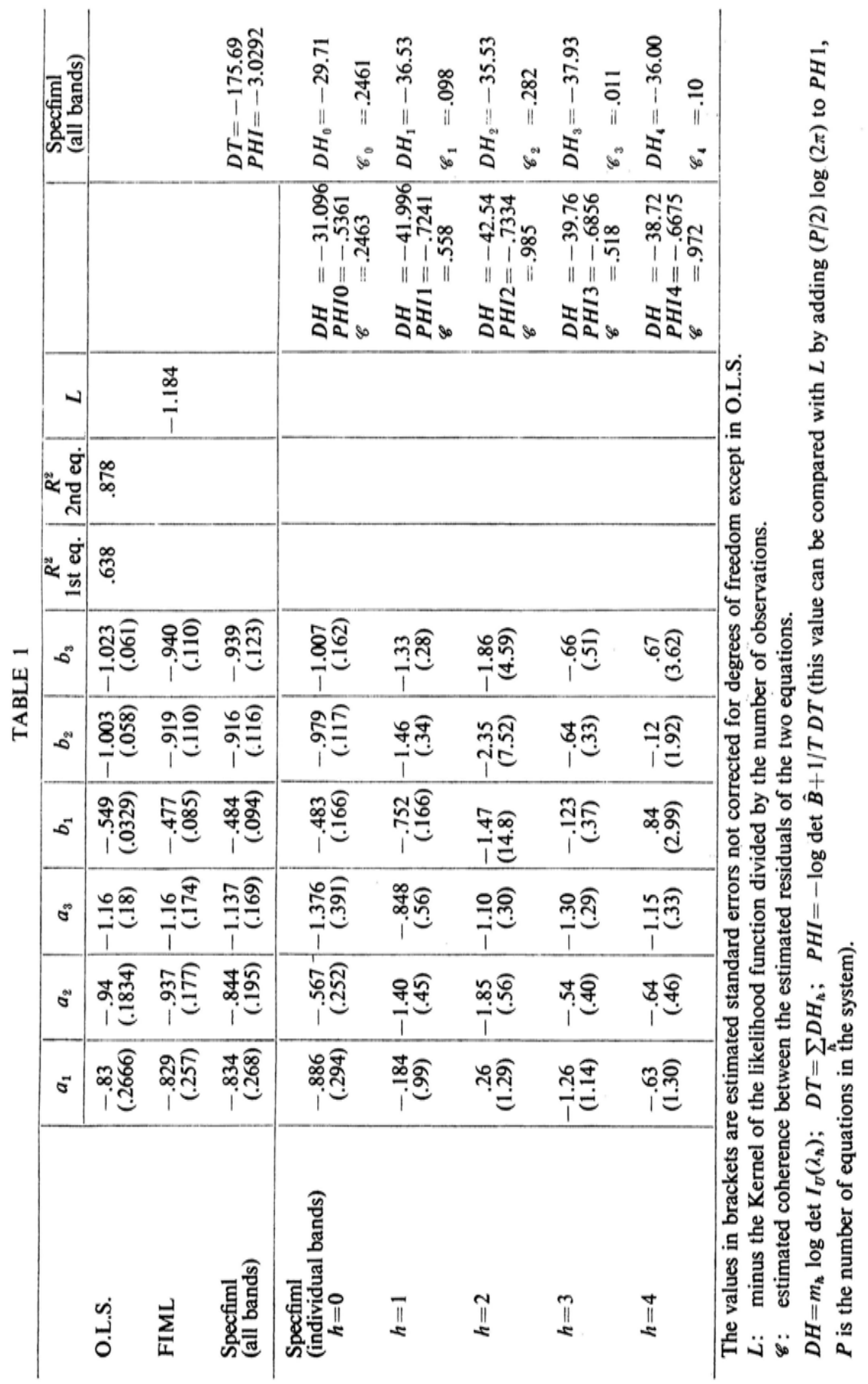


TABLE 2

THE SPECTRAL ESTIMATION OF THE INFLATION MODEL, FOR THE OVER-ALL AND INDIVIDUAL FREQUENCY BANDS

Wage Equation

\begin{tabular}{|c|c|c|c|c|}
\hline \multirow[b]{2}{*}{ Variable } & \multicolumn{4}{|c|}{ Specfiml Estimates Using: } \\
\hline & $\begin{array}{c}\text { All Frequency } \\
\text { Bands }\end{array}$ & $\begin{array}{c}\text { Frequency Band } \\
\text { Around Zero }\end{array}$ & $\begin{array}{c}\text { Frequency Band } \\
\text { Around } \pi / 2\end{array}$ & $\begin{array}{l}\text { Frequency Band } \\
\text { Around } \pi\end{array}$ \\
\hline$\Delta U$ & $\begin{array}{l}-.023 \\
(1.71)\end{array}$ & $\begin{array}{l}-.016 \\
(1.49)\end{array}$ & $\begin{array}{r}-.003 \\
(.74)\end{array}$ & $\begin{array}{r}-.005 \\
(.95)\end{array}$ \\
\hline$R W_{-1}$ & $\begin{array}{l}-.37 \\
(2.09)\end{array}$ & $\begin{array}{l}-.10 \\
(.56)\end{array}$ & $\begin{array}{r}-1.03 \\
(1.74)\end{array}$ & $\begin{array}{l}-.25 \\
(6.87)\end{array}$ \\
\hline$R E_{-1}$ & $\begin{array}{c}.29 \\
(2.12)\end{array}$ & $\begin{array}{l}.12 \\
(.62)\end{array}$ & $\begin{array}{c}.75 \\
(1.53)\end{array}$ & $\begin{array}{c}.18 \\
(6.99)\end{array}$ \\
\hline$S P_{-1}$ & $\begin{array}{c}.01 \\
(2.92)\end{array}$ & $\begin{array}{l}.001 \\
(.37)\end{array}$ & $\begin{array}{r}.012 \\
(4.25)\end{array}$ & $\begin{array}{l}.001 \\
(.47)\end{array}$ \\
\hline$T 1$ & $\begin{array}{l}-.0032 \\
(4.96)\end{array}$ & $\begin{array}{l}-.0019 \\
(16.68)\end{array}$ & $\begin{array}{c}-.0026 \\
(.66)\end{array}$ & $\begin{array}{l}.002 \\
(.29)\end{array}$ \\
\hline$T 1^{2}$ & $\begin{array}{l}.00003 \\
(4.19)\end{array}$ & $\begin{array}{l}.00003 \\
(4.78)\end{array}$ & $\begin{array}{l}.00003 \\
(.23)\end{array}$ & $\begin{array}{l}.00002 \\
(.15)\end{array}$ \\
\hline$\Delta W_{-1}$ & $\begin{array}{c}.18 \\
(1.34)\end{array}$ & $\begin{array}{c}.34 \\
(1.97)\end{array}$ & $\begin{array}{l}.04 \\
(.15)\end{array}$ & $\begin{array}{l}-1.52 \\
(12.54)\end{array}$ \\
\hline$\Delta W_{-2}$ & $\begin{array}{l}-.53 \\
(4.03)\end{array}$ & $\begin{array}{l}.22 \\
(.40)\end{array}$ & $\begin{array}{l}-.53 \\
(4.67)\end{array}$ & $\begin{array}{r}-1.10 \\
(3.52)\end{array}$ \\
\hline$\Delta W_{-3}$ & $\begin{array}{c}.23 \\
(2.32)\end{array}$ & $\begin{array}{l}-.45 \\
(1.67)\end{array}$ & $\begin{array}{c}.06 \\
(.29)\end{array}$ & $\begin{array}{r}-.20 \\
(.83)\end{array}$ \\
\hline$R W_{-3}$ & $\begin{array}{l}-.44 \\
(2.99)\end{array}$ & $\begin{array}{c}.27 \\
(1.73)\end{array}$ & $\begin{array}{l}-.65 \\
(2.90)\end{array}$ & $\begin{array}{l}.035 \\
(.47)\end{array}$ \\
\hline$R E_{-3}$ & $\begin{array}{c}.35 \\
(3.79)\end{array}$ & $\begin{array}{l}.17 \\
(.89)\end{array}$ & $\begin{array}{c}.38 \\
(1.94)\end{array}$ & $\begin{array}{l}-.07 \\
(1.56)\end{array}$ \\
\hline
\end{tabular}

Earnings Equation

\begin{tabular}{l|c|c|c|c}
\hline \multirow{3}{*}{ Variable } & \multicolumn{4}{|c}{ Specfiml Estimates Using: } \\
\cline { 2 - 5 }$\Delta W$ & $\begin{array}{c}\text { All Frequency } \\
\text { Bands }\end{array}$ & $\begin{array}{c}\text { Frequency Band } \\
\text { Around Zero }\end{array}$ & $\begin{array}{c}\text { Frequency Band } \\
\text { Around } \pi / 2\end{array}$ & $\begin{array}{c}\text { Frequency Band } \\
\text { Around } \pi\end{array}$ \\
\cline { 2 - 5 }$R W_{-1}$ & .86 & .74 & 3.06 & .30 \\
& $(4.62)$ & $(3.75)$ & $(3.10)$ & $(1.27)$ \\
$R E_{-1}$ & .82 & .28 & .09 & 1.60 \\
$T 1$ & $(3.79)$ & $(1.83)$ & $(.13)$ & $(4.42)$ \\
& -.67 & -.23 & -1.38 & -1.82 \\
$\Delta W_{-2}$ & $(3.80)$ & $(1.84)$ & $(2.50)$ & $(15.24)$ \\
& .0022 & .0009 & .014 & .011 \\
$\Delta W_{-4}$ & $(4.00)$ & $(2.25)$ & $(2.93)$ & $(5.53)$ \\
$\left(P_{-1}-P_{-4}\right)$ & -.53 & -.16 & 2.10 & .61 \\
& $(3.81)$ & $(.69)$ & $(1.54)$ & $(3.18)$ \\
$S P_{-5}$ & -.42 & -.21 & .50 & -.47 \\
& $(3.78)$ & $(.94)$ & $(.86)$ & $(2.81)$ \\
& .35 & .09 & -.48 & .08 \\
& $(4.30)$ & $(1.09)$ & $(1.20)$ & $(.67)$ \\
& .004 & -.002 & -.013 & .006 \\
& $(.77)$ & $(.44)$ & $(1.38)$ & $(.96)$ \\
\hline
\end{tabular}


TABLE 2

(Continued)

Price Equation

\begin{tabular}{l|c|c|c|c}
\hline \multirow{3}{*}{ Variable } & \multicolumn{4}{|c}{ Specfiml Estmates Using: } \\
\cline { 2 - 5 }$\Delta E$ & $\begin{array}{c}\text { All Frequency } \\
\text { Bands }\end{array}$ & $\begin{array}{c}\text { Frequency Band } \\
\text { Around Zero }\end{array}$ & $\begin{array}{c}\text { Frequency Band } \\
\text { Around } \pi / 2\end{array}$ & $\begin{array}{c}\text { Frequency Band } \\
\text { Around } \pi\end{array}$ \\
\cline { 2 - 5 }$R E_{-1}$ & .24 & .58 & -.06 & .55 \\
$(1.53)$ & $(2.11)$ & $(.28)$ & $(2.48)$ \\
$\left(P M_{-1}-P_{-1}\right)$ & .47 & .17 & .80 & 1.68 \\
$T$ & $(4.20)$ & $(1.83)$ & $(1.85)$ & $(5.77)$ \\
& .11 & .05 & .04 & -.11 \\
$R E_{-3}$ & $(5.54)$ & $(2.84)$ & $(.57)$ & $(1.06)$ \\
$\left(P_{-1}-P_{-4}\right)$ & -.004 & -.004 & -.009 & -.007 \\
& $(5.16)$ & $(6.31)$ & $(1.92)$ & $(2.03)$ \\
& .15 & .43 & .47 & -.62 \\
& $(2.35)$ & $(3.05)$ & $(2.10)$ & $(2.86)$ \\
\hline
\end{tabular}

Unemployment Equation

\begin{tabular}{l|c|c|c|c}
\hline \multirow{2}{*}{ Variable } & \multicolumn{4}{|c}{ Specfiml Estimates Using: } \\
\cline { 2 - 5 }$U_{-2}$ & $\begin{array}{c}\text { All Frequency } \\
\text { Bands }\end{array}$ & $\begin{array}{c}\text { Frequency Band } \\
\text { Around Zero }\end{array}$ & $\begin{array}{c}\text { Frequency Band } \\
\text { Around } \pi / 2\end{array}$ & $\begin{array}{c}\text { Frequency Band } \\
\text { Around } \pi\end{array}$ \\
\hline$G D P$ & -.37 & -.34 & -.88 & 1.18 \\
$T$ & $-7.45)$ & $(6.57)$ & $(6.20)$ & $(3.28)$ \\
& -3.9 & -4.2 & -2.7 & -1.8 \\
& $(6.13)$ & $(6.08)$ & $(2.57)$ & $(1.44)$ \\
\hline$P H I$ & $(6.51)$ & $(6.39)$ & $(3.63)$ & .004 \\
$D H_{0}$ & -20.235 & -6.40 & -8.62 & -7.39 \\
$D H_{1}$ & -514.17 & -537.77 & & \\
$D H_{2}$ & -678.70 & & -724.21 & -620.46 \\
$m_{0}$ & -506.88 & & & \\
$m_{1}$ & 24 & 24 & 16 & 24 \\
$m_{2}$ & 16 & & & \\
\hline
\end{tabular}

Description of the data:

All the data have been previously deseasonalized by a multiple regression with a constant and three quarterly dummies (i. e., the periodogram ordinates for $\omega_{j}, j=0, T / 4, T / 2$ and $3 T / 4$ have been omitted for all the variables).

All the variables except $G P D$ are expressed in index numbers based on average $1948=100$. All variables enter the equations in logs.

If $X$ is a variable in the system

$$
\begin{aligned}
& \Delta X=X-L X, \text { where } L \text { is the back operator } \\
& X_{-j}=L^{j} X
\end{aligned}
$$

$W$ official weekly wage-rate index

$E$ weekly earnings per employee in employment

$R W=W-P$

$R E=E-P$

$P$ consumers' price deflator

$P M$ official import price index 
TABLE 2

(Continued)
$G D P$ real GDP
$U$ index number of total unemployment
$S P$ number of industrial stoppages in all industries except coal mining
$T$ trend variable, $1949(2)=1, \ldots, 1971(1)=88$
$T 1$ trend variable, $1950(2)=\cdots=1958(4)=0,1959(1)=1, \ldots, 1971(1)=49$

Notes: Values in brackets are asymptotic $t$ values, not corrected for degrees of freedom.

See Table 1 for definition of $\mathrm{PHi}, \mathrm{DHh}, \ldots$.

This, perhaps, is not surprising, since for that model a first order AR process for the errors is rejected according to results in Espasa [3].

Looking at the results from individual bands estimation we have that the estimates of some coefficients are significantly different for different bands. This can be due to the fact that the model has been designed without distinguishing between slowly-changing and rapidly-changing situations in the sample period, or it can be reflecting that the time unit used and the restrictions imposed are a bad approximation of the underlying differential system. In any case it seems that the estimation of a model by individual bands can be a useful instrument to detect misspecifications that cannot be easily observed from time domain methods. From our results here, these misspecifications are more likely to occur on the dynamic elements of an aggregate model.

\section{REFERENCES}

[1] EspasA, A., "The Spectral Estimation of the Simultaneous Equations Model and its Relation with Time Domain Methods," revised and enlarged version of a paper presented at the European Meeting of the Econometric Society, Grenoble 1974.

[2] — "The Estimation of the Multiple Regression Model with Stationary Errors and Lagged Endogenous Variables," unpublished paper, London School of Economics, March 1975.

[3] — - "A Wages-Earning-Prices Inflation Model for United Kingdom 1950-70: Its Specification and Estimation by Classical and Spectral Methods," unpublished Ph. D. thesis, London University, 1975.

[4] Hannan, E. J., Multiple Time Series (New York: John Wiley, 1970).

[ 5 [ — , "The Identification Problem for Multiple Equation Series with Moving Average Errors," Econometrica, XXXIX (September, 1971), 751-766.

[6] - AND D. F. Nicholls, "The Estimation of Mixed Regression, Autoregression, Moving Average, and Distributed Lag Models," Econometrica, XL, (May, 1972), 529.548.

[7] - and R. D. Terrell, "Multiple Equation Systems with Stationary Errors," Econometrica, XLI, (March, 1973), 299-320.

[ 8 ] Hendry, D. F., "Users' Manual for FIML, Full Information Maximum Likelihood Estimation of Systems of Linear Equations," London School of Economics, 1972.

[9] —_ "Users' Manual for ARFIML, Full Information Maximum Likelihood Estimation of Linear Equations with Errors Generated by a First Order Vector Autoregressive Process," London School of Economics, 1972. 
[10] Nicholls, D. F., "The Efficient Estimation of Vector Linear Time Series Models," unpublished paper, Australian National University and Michigan State University, 1975.

[11] Powell, M. J. D., "An Efficient Method for Finding the Minimum of a Function of Several Variables without Calculating Derivatives," Computer Journal, VII (1964), 303-307.

[12] Sargan, J. D., "The Identification and Estimation of Sets of Simultaneous Stochastic Equations," unpublished paper, London School of Economics, 1972. 